\title{
Decomposing a Matrix into two Submatrices with Extremely Small Operator Norm
}

\author{
I.V.Limonova* \\ Lomonosov Moscow State University
}

\begin{abstract}
We give sufficient conditions on a matrix A ensuring the existence of a partition of this matrix into two submatrices with extremely small norm of the image of any vector. Under some weak conditions on a matrix A we obtain a partition of A with the extremely small $(1, q)-$ norm of submatrices.
\end{abstract}

Keywords: submatrix, operator norm, partition of a matrix, Lunin's method

This paper is devoted to the estimates of operator norms of submatrices. The subject is actively being developed and finds various applications. The present work can be viewed as a continuation of [1] discussing the $(2,1)$-norm case. This case was studied earlier for matrices with orthonormal columns in [2], where an analogue of the partition (2) (see below) with the extremely small $(2,1)-$ norm of the corresponding submatrices was obtained. Using the modified Lunin's method we prove an essential reinforcement of Assertion 4 from [1] and the generalization of Assertion 3 to the case of the $(X, q)$-norm with $1 \leq q<\infty$. We study the case of the $(1, q)$-norm in greater detail.

For an $N \times n$ matrix $A$, viewed as an operator from $l_{p}^{n}$ to $l_{q}^{N}$, we define the $(p, q)$-norm:

$$
\|A\|_{(p, q)}=\sup _{\|x\|_{l_{p}} \leq 1}\|A x\|_{l_{q}^{N}}, 1 \leq p, q \leq \infty .
$$

In fact, Proposition 2 is proved here for a more general $(X, q)$-norm where $X$ is an $n$-dimensional norm space.

We use the following notation: $\operatorname{rk}(A)$ is the rank of a matrix $A,\langle N\rangle$ is the set of natural numbers $1,2, \ldots, N ; v_{i}, i \in\langle N\rangle$ stands for the rows of $A, w_{j}, j \in\langle n\rangle-$ its columns. For a subset $\omega \subset\langle N\rangle$ $A(\omega)$ denotes the submatrix of a matrix $A$ formed by the rows $v_{i}, i \in \omega, \bar{\omega}=\langle N\rangle \backslash \omega$. $(\cdot, \cdot)$ stands for the inner product in $\mathbb{R}^{n},\|x\|_{p}$ is the norm of $x \in \mathbb{R}^{n}$ in $l_{p}^{n}, 1 \leq p \leq \infty$. For a norm space $X\|\cdot\|_{X}$ is the norm on $\mathrm{X}$.

The following condition is the counterpart of the condition on a matrix from [1] in the case of an arbitrary $1 \leq q<\infty$ :

$$
\forall x \in \mathbb{R}^{n} \quad \forall i_{0} \in\langle N\rangle \quad\left|\left(v_{i_{0}}, x\right)\right| \leq \varepsilon\left(\sum_{i=1}^{N}\left|\left(v_{i}, x\right)\right|^{q}\right)^{1 / q} .
$$

*e-mail: limonova_irina@rambler.ru. 
Proposition 1. Assume that an $N \times n$ matrix A stisfies (1) with $0<\varepsilon \leq(\operatorname{rk}(A))^{-1 / q}$ and $1 \leq q<\infty$. Then there exists a partition

$$
\langle N\rangle=\Omega_{1} \cup \Omega_{2}, \Omega_{1} \cap \Omega_{2}=\emptyset,
$$

such that

$$
\left\|A\left(\Omega_{k}\right) x\right\|_{q} \leq \gamma\|A x\|_{q}, \quad \gamma=\frac{1}{2^{1 / q}}+\frac{2+3 \cdot 2^{-1 / q}}{q}\left(\operatorname{rk}(A) \varepsilon^{q} \ln \frac{6 q}{\left(\operatorname{rk}(A) \varepsilon^{q}\right)^{1 / 3}}\right)^{1 / 3}
$$

for any $x \in \mathbb{R}^{n}$ and $k=1,2$.

Remark 1. No one knows whether such a partition exists or not if $1<\operatorname{rk}(A) \varepsilon^{q}$.

Sketch of proof. First, we prove Proposition 1 for the case of $\operatorname{rk}(A)=n$. Denote

$$
\delta=\frac{\left(n \varepsilon^{q}\right)^{1 / 3}}{q} .
$$

Let $X$ be the space $\mathbb{R}^{n}$ with the norm $\|x\|_{X}=\|A x\|_{q}$ (it is a norm on $\mathbb{R}^{n}$ because $\left.\operatorname{rk}(A)=n\right)$. Let $S_{X}=\left\{x \in \mathbb{R}^{n}:\|x\|_{X}=1\right\}$ be the unit sphere of $X$. Let $\mathbb{Y}$ be a $\delta$-net in the norm $\|\cdot\|_{X}$ on the sphere $S_{X}$ with at most $\leq(3 / \delta)^{n}$ elements. Suppose that it is not the case, then for every partition (2) there exists a vector $x_{1} \in S_{X}$ such that

$$
\left\|A\left(\Omega_{1}\right) x_{1}\right\|_{q}>\gamma\left\|A x_{1}\right\|_{q}
$$

(in this case let $\omega^{\prime}=\Omega_{1}, x_{\omega^{\prime}}=x_{1}$ ), or there exists a vector $x_{2} \in S_{X}$ such that

$$
\left\|A\left(\Omega_{2}\right) x_{2}\right\|_{q}>\gamma\left\|A x_{2}\right\|_{q}
$$

(then we define $\left.\omega^{\prime}=\Omega_{2}, x_{\omega^{\prime}}=x_{2}\right)$. For every pair $\left(\Omega_{1}, \Omega_{2}\right)$ we find $\omega^{\prime}$ and $x_{\omega^{\prime}}$. Let $y_{\omega^{\prime}}$ be one of the nearest to $x_{\omega^{\prime}}$ vectors from the net $\mathbb{Y}$. There are $2^{N-1}-1$ different partitions of the set $\langle N\rangle$ into two nonempty parts. Therefore there exists a vector $y_{0} \in \mathbb{Y}$ such that the set $K=\left\{\omega^{\prime}: y_{0}=y_{\omega^{\prime}}\right\}$ is large enough:

$$
|K| \geq\left(2^{N-1}-1\right)\left(\frac{\delta}{3}\right)^{n} \geq 2^{N}\left(\frac{\delta}{6}\right)^{n}
$$

(Here we assume that $n>1$, otherwise Proposition 1 is obvious.) Therefore there is a vector $y_{0} \in S_{X}$ and at least $2^{N}(\delta / 6)^{n}$ subsets $\omega^{\prime} \subset\langle N\rangle$ for which $\left\|A\left(\omega^{\prime}\right) x_{\omega^{\prime}}\right\|_{q}>\gamma\left\|A x_{\omega^{\prime}}\right\|_{q}$ and $\left\|y_{0}-x_{\omega^{\prime}}\right\|_{X}<\delta$. Note that for $x \in S_{X}$ and $\omega \subset\langle N\rangle\|A(\omega) x\|_{q} \leq\|A(\omega)\|_{(X, q)} \leq\|A\|_{(X, q)}$.

Below we assume that $\gamma<1$, otherwise (3) is obviously true. As $\gamma<1$, for $\omega^{\prime} \in K$ we obtain:

$$
\begin{gathered}
\left\|A\left(\omega^{\prime}\right) y_{0}\right\|_{q} \geq\left\|A\left(\omega^{\prime}\right) x_{\omega^{\prime}}\right\|_{q}-\left\|A\left(\omega^{\prime}\right)\left(x_{\omega^{\prime}}-y_{0}\right)\right\|_{q}> \\
>\gamma\left\|A x_{\omega^{\prime}}\right\|_{q}-\delta\left\|A\left(\omega^{\prime}\right)\left\{\frac{x_{\omega^{\prime}}-y_{0}}{\left\|x_{\omega^{\prime}}-y_{0}\right\|_{X}}\right\}\right\|_{q} \geq\left(\gamma\left\|A y_{0}\right\|_{q}-\gamma\left\|A\left(x_{\omega^{\prime}}-y_{0}\right)\right\|_{q}\right)- \\
-\delta\left\|A\left(\frac{x_{\omega^{\prime}}-y_{0}}{\left\|x_{\omega^{\prime}}-y_{0}\right\|_{X}}\right)\right\|_{q} \geq \gamma\left\|A y_{0}\right\|_{q}-2 \delta \geq \gamma\left\|A y_{0}\right\|_{q}-2 \delta\left\|A y_{0}\right\|_{q}=\left\|A y_{0}\right\|_{q}(\gamma-2 \delta) .
\end{gathered}
$$

Since $y_{0} \in S_{X}$, we have used $\left\|A y_{0}\right\|_{q}=\left\|y_{0}\right\|_{X}=1$ in the last inequality. Let $R$ be an amount of subsets $\omega \subset\langle N\rangle$ for which

$$
\left\|A(\omega) y_{0}\right\|_{q} \geq(\gamma-2 \delta)\left\|A y_{0}\right\|_{q}
$$


holds. Let $K_{1}$ be the set of such subsets. Let us show that $R<2^{N}(\delta / 6)^{n}$, then we will come to the contradiction, and it will complete the proof of Proposition 1 in the case of $\operatorname{rk}(A)=n$. Denote $M=3 \cdot 2^{-1 / q}$. Since $\delta \leq \phi(n, \varepsilon)$, then for $\omega^{\prime} \in K_{1}$ we have:

$$
\begin{gathered}
\sum_{i \in \omega^{\prime}}\left|\left(v_{i}, y_{0}\right)\right|^{q}>(\gamma-2 \delta)^{q} S>\left(\frac{1}{2^{1 / q}}+M \phi(n, \varepsilon)\right)^{q} S \geq \\
\geq\left(\frac{1}{2}+q \frac{1}{2^{(q-1) / q}} M \phi(n, \varepsilon)\right) S=\left(\frac{1}{2}+q \frac{2^{1 / q}}{2} M \phi(n, \varepsilon)\right) S .
\end{gathered}
$$

$R$ can be estimated as in the proof of Assertion 3 from 1 .

Now, let a matrix have the rank $r<n$. Without loss of generality, we can assume that the vectors $w_{1}, \ldots, w_{r}$ are linearly independent. It is clear that (1) holds for the matrix $\tilde{A}$, which consists from the first $r$ columns of $A$. We have $\operatorname{rk} \tilde{A}=r$, therefore there exists a partition of the form (2) such that (3) holds. Let $w_{j}=\sum_{i=1}^{r} \lambda_{j}^{i} w_{i}$. For a vector $x \in \mathbb{R}^{n}$ we construct the vector $\tilde{x} \in \mathbb{R}^{r}$ having coordinates $\tilde{x}_{i}=x_{i}+\sum_{j=r+1}^{n} \lambda_{j}^{i} x_{j}$, then $A x=\tilde{A} \tilde{x}$ and for $k=1,2 A\left(\Omega_{k}\right) x=\tilde{A}\left(\Omega_{k}\right) \tilde{x}$, so for the partition we have found (3) also holds for the matrix $A$.

Corollary 1. Assume that an $N \times n$ matrix A satisfies (1) with $0<\varepsilon \leq(\operatorname{rk}(A))^{-1 / q}$ and $1 \leq q<\infty$. Then there exists a partition (2) such that for any $x \in \mathbb{R}^{n}$ and $k=1,2$ we have

$$
\left(\frac{1}{2}-\psi\right) \sum_{i \in \Omega_{k}}\left|\left(v_{i}, x\right)\right|^{q} \leq \sum_{i=1}^{N}\left|\left(v_{i}, x\right)\right|^{q} \leq\left(\frac{1}{2}+\psi\right) \sum_{i \in \Omega_{k}}\left|\left(v_{i}, x\right)\right|^{q},
$$

where

$$
\psi=2^{q+1}\left(\operatorname{rk}(A) \varepsilon^{q} \ln \frac{6 q}{\left(\operatorname{rk}(A) \varepsilon^{q}\right)^{1 / 3}}\right)^{1 / 3} .
$$

The following proposition is a simple corollary of Proposition 1 .

Proposition 2. Let for an $N \times n$ matrix $A$ (1) hold for some $0<\varepsilon \leq(\operatorname{rk}(A))^{-1 / q}$ and $1 \leq q<\infty$. Then there exists a partition (2) such that for $k=1,2$ the following inequality holds

$$
\left\|A\left(\Omega_{k}\right)\right\|_{(X, q)} \leq \gamma\|A\|_{(X, q)}
$$

where $\gamma$ is defined in the formulation of Proposition 1 .

The following proposition is analogous to Proposition 2 for the $(1, q)$-norm. Let $e_{j}, j \in\langle n\rangle$ be the standard basis in $\mathbb{R}^{n}$.

Proposition 3. If for an $N \times n$ matrix $A$ the inequality

$$
\left|a_{j}^{i}\right| \leq \varepsilon\left\|w_{j}\right\|_{q}
$$

holds for some $1 \leq q<\infty$ and $0<\varepsilon<1$ and for every $i \in\langle N\rangle$, and $j \in\langle n\rangle$, then there exists a partition (2) such that for $k=1,2$ the following holds:

a) $\left\|A\left(\Omega_{k}\right)\right\|_{(1, q)} \leq\left(\frac{1}{2}+\frac{3}{2} \varepsilon^{q / 3} \ln ^{1 / 3}(4 n)\right)^{1 / q}\|A\|_{(1, q)}$,

b) $\left\|A\left(\Omega_{k}\right)\right\|_{(1, q)} \leq\left(\frac{1}{2}+\frac{1}{2} \varepsilon^{q} \sqrt{N}\left(1+\log \left(\frac{n}{N}+1\right)^{1 / 2}\right)^{1 / q}\|A\|_{(1, q)}\right.$,

c) $\left\|A\left(\Omega_{k}\right)\right\|_{(1, q)} \leq\left(\frac{1+n \varepsilon^{q}}{2}\right)^{1 / q}\|A\|_{(1, q)}$. 
Remark 2. In Proposition 3 we need sufficiently weak conditions (compared to Proposition 1) on the elements of a matrix.

Proof. Since the function $\|A x\|_{q}$ is convex, then the $(1, q)$-norm of a matrix is attained on one of the vectors from the standard basis.

The proof of a) is indeed close to the previous arguments from Proposition 1, so here we only show the sketch. Assume that our proposition is not true and for each partition (2) there exists a number $k$ such that $\left\|A\left(\Omega_{k}\right)\right\|_{(1, q)}>\left(1 / 2+(3 / 2) \varepsilon^{q / 3} \ln ^{1 / 3}(4 n)\right)^{1 / q}\|A\|_{(1, q)}$. Denote $\omega^{\prime}=\Omega_{k}$. The $(1, q)$-norm of the matrix $A_{\omega^{\prime}}$ is attained on some vector $e_{j_{\omega^{\prime}}}, j_{\omega^{\prime}} \in\langle n\rangle$, therefore the following holds: $\sum_{i \in \omega^{\prime}}\left|a_{j_{\omega^{\prime}}}^{i}\right|^{q}>\left(1 / 2+(3 / 2) \varepsilon^{q / 3} \ln ^{1 / 3}(4 n)\right)\left\|w_{j_{\omega^{\prime}}}\right\|^{q}$. Like in the proof of Proposition 1, there exists $j_{0} \in\langle n\rangle$ such that the set $K=\left\{\omega^{\prime}: j_{\omega^{\prime}}=j_{0}\right\}$ is large enough:

$$
|K| \geq\left(2^{N-1}-1\right) / n>2^{N-2} / n
$$

It is easy to see that for every $\omega \in K$

$$
\sum_{i \in \omega}\left|a_{j_{0}}^{i}\right|^{q}>\left(1 / 2+(3 / 2) \varepsilon^{q / 3} \ln ^{1 / 3}(4 n)\right)\left\|w_{j_{0}}\right\|^{q} .
$$

So, for the proof of a) it is enough to check that a number $R$ of subsets $\omega \subset\langle N\rangle$ for which (7) holds is less than the right part of (6). The value $R$ is estimated as in the proof of Assertion 3 from [1].

To prove b) we use Corollary 5 from [3].

Let $\tilde{w}_{j}=\left(\left|a_{j}^{1}\right|^{q}, \ldots,\left|a_{j}^{N}\right|^{q}\right)$ be a vector which is obtained from the $j$-th column of $A$ by raising the moduli of its coordinates to the power $q$. For all $j \in\langle n\rangle\left\|w_{j}\right\|_{q}^{q} \leq\|A\|_{(1, q)}$, so (5) implies that $\left\|\tilde{w}_{j}\right\|_{\infty} \leq \varepsilon^{q}\|A\|_{(1, q)}^{q}$. Then due to Corollary from [3] mentioned above there exists such a vector $\xi=\left(\xi_{1}, \ldots, \xi_{N}\right) \in \mathbb{R}^{N}$, whose coordinates have modulus 1 such that for every $j \in\langle n\rangle$ the following inequality holds:

$$
\left|\left(\tilde{w}_{j}, \xi\right)\right| \leq \varepsilon^{q} \sqrt{N}\left(1+\log \left(\frac{n}{N}+1\right)\right)^{1 / 2}\|A\|_{(1, q)}^{q} .
$$

Let $\Omega_{1}=\left\{i \in\langle N\rangle: \xi_{i}=1\right\}, \Omega_{2}=\langle N\rangle \backslash \Omega_{1}=\left\{i \in\langle N\rangle: \xi_{i}=-1\right\}$. Let us check b). Denote $\theta=\sqrt{N}\left(1+\log \left(\frac{n}{N}+1\right)\right)^{1 / 2}$. For $k=1,2$ there exists $j_{0}^{k} \in\langle n\rangle$ such that

$$
\left\|A\left(\Omega_{k}\right)\right\|_{(1, q)}^{q}=\sum_{i \in \Omega_{k}}\left|a_{j_{0}^{k}}^{i}\right|^{q} \leq \frac{1}{2}\left(\sum_{i \in\langle N\rangle}\left|a_{j_{0}}^{i}\right|^{q}+\varepsilon^{q} \theta\|A\|_{(1, q)}^{q}\right) \leq\left(\frac{1}{2}+\frac{1}{2} \varepsilon^{q} \theta\right)\|A\|_{(1, q)}^{q},
$$

as required.

To prove c) we apply the following theorem.

Theorem ([4], p. 287). Let $A_{1}, \ldots, A_{n}$ be sets in $\mathbb{R}^{n}$ with finite Lebesgue measure, then there exists a hyperplane $\pi$ which divides the measure of each of them in half.

Let $M=\max _{i, j}\left\{\left|a_{j}^{i}\right|^{q}\right\}+1$. One can put in $\mathbb{R}^{n} N$ cubes with sides equal to $M$ and parallel to the axes such that every hyperplane intersects at most $n$ of them. (It follows from the existence of $N$ points of the general position in $\mathbb{R}^{n}$ and the continuity of the equation of a plane.) Let us numerate these cubes. For $i \in\langle N\rangle$ let $u_{i}$ be the vertex of $i$-th cube with the smallest coordinates. For each entry $a_{j}^{i}$ of the matrix we define a parallelepiped $\widetilde{P_{j}^{i}}=[0,1]^{j-1} \times\left[1,1+\left|a_{j}^{i}\right|^{q}\right] \times[0,1]^{n-j}$. We put $n$ 
rectangular parallelepipeds defined by the entries of the row $v_{i}\left(P_{j}^{i}=u_{i}+\widetilde{P_{j}^{i}}\right)$ into the cube with number $i$. Note that $\mu\left(P_{j}^{i}\right)=\left|a_{j}^{i}\right|^{q}$. We call the set of $P_{j}^{i}, j \in\langle n\rangle$ for a fixed $i$ by an $i$-th "angle".

For $j \in\langle n\rangle$ let $A_{j}=\bigcup_{i \in\langle N\rangle} P_{j}^{i}$. Applying the theorem mentioned above to $A_{j}$, we get a hyperplane $\pi$ which divides in half the measure of each $A_{j}$. Let $P_{1}$ and $P_{2}$ be halfspaces into which $\pi$ divides $\mathbb{R}^{n}$. By construction $\pi$ intersects at most $n$ cubes, consequently at most $n$ "angles". It is clear now how to obtain a partition (2). We put the indices of the "angles" which entirely belong to $P_{1}\left(\right.$ or $\left.P_{2}\right)$ in $\Omega_{1}$ (in $\Omega_{2}$ correspondingly). We put the indices of the "angles" which intersect both $P_{1}$ and $P_{2}$ in $\Omega_{1}$. Let $G$ be the set of such indices. Let us show that for every $j \in\langle n\rangle$ the $l_{q}^{N}$-norm of the column $w_{j}$ will decrease at least $\left(\frac{1+n \varepsilon^{q}}{2}\right)^{1 / q}$ times under the partition. It will prove our proposition. Since $\pi$ divides in half the measure of $A_{j}$, then

$$
\sum_{i \in \Omega_{1} \backslash G}\left|a_{j}^{i}\right|^{q}+V_{1}=\sum_{i \in \Omega_{2}}\left|a_{j}^{i}\right|^{q}+V_{2}
$$

where $V_{k}, k=1,2$ stands for the volume of $\cup_{i \in\langle G\rangle} P_{j}^{i} \cap P_{k}$. From (5) and due to the fact that $\pi$ intersects at most $n$ "angles", we have the following inequality:

$$
V_{1}+V_{2} \leq n \varepsilon^{q} \sum_{i \in\langle N\rangle}\left|a_{j}^{i}\right|^{q}
$$

so for $k=1,2$

$$
\sum_{i \in \Omega_{k}}\left|a_{j}^{i}\right|^{q} \leq \frac{1+n \varepsilon^{q}}{2} \sum_{i \in\langle N\rangle}\left|a_{j}^{i}\right|^{q} .
$$

Thus, Proposition 3 is proved.

The following proposition shows that there is a case when (5) holds for $\varepsilon<1$ but for every partition one of the submatrices has the same $(1, q)$-norm as the whole matrix.

Proposition 4. For $n=2^{2 k-1}$ there exists a $2 k \times n$ - matrix A for which (5) holds for $\varepsilon^{q} \log _{2} 2 n \geq 2$, but for every partition of the form (2) the following equality holds:

$$
\max \left\{\left\|A\left(\Omega_{1}\right)\right\|_{(1, q)},\left\|A\left(\Omega_{2}\right)\right\|_{(1, q)}\right\}=\|A\|_{(1, q)} .
$$

Sketch of proof. For every pair of the subsets $\omega$ and $\langle 2 k\rangle \backslash \omega$ of the set $\langle 2 k\rangle$ we choose the subset (any) of the largest cardinality. Let us numerate such subsets: $B_{1}, \ldots, B_{2^{2 k-1}}$. We construct a matrix $A$ in the following way: if $i \in B_{j}$, then $a_{j}^{i}=\frac{1}{\left|B_{j}\right|^{1 / q}}$, otherwise, $a_{j}^{i}=0$. It is easy to check that (5) holds for $A$ and that for any partition (2) either $\left\|A\left(\Omega_{1}\right)\right\|_{(1, q)}=\|A\|_{(1, q)}$ or $\left\|A\left(\Omega_{2}\right)\right\|_{(1, q)}=\|A\|_{(1, q)}$.

Let $q=\infty$ and $A$ be an arbitrary matrix. There is no partition that decreases (even a little) $(X, \infty)$-norms of two submatrices. It is because one can find a row $v_{\text {sup }}$ of the matrix $A$ such that $\|A\|_{(X, \infty)}=\sup _{\|x\|_{X} \leq 1}\left\langle x, v_{\text {sup }}\right\rangle$, and then the norm of the submatrix containing a row $v_{\text {sup }}$, will be equal to the norm of $A$.

The work was supported by the Russian Federation Government Grant No. 14.W03.31.0031. The paper is submitted to Mathematical Notes. 


\section{References}

[1] B. S. Kashin, I. V. Limonova, "Decomposing a Matrix into two Submatrices with Extremally Small (2,1)-Norm", Math. Notes, 106:1 (2019), 63-70

[2] B. S. Kashin, "Decomposing an orthogonal matrix into two submatrices with extremally small (2,1)-norm", Russian Math. Surveys, 72:5 (2017), 971-973

[3] E. D. Gluskin, "Extremal properties of orthogonal parallelepipeds and their applications to the geometry of Banach spaces", Math. USSR-Sb., 64:1 (1989), 85-96

[4] B. Gray, Homotopy theory, Academic Press, New York, San Francisco, London 1975 